\title{
NOISE WAVE ANALYSIS OF DICKE AND NOISE INJECTION RADIOMETERS: COMPLETE S-PARAMATER ANALYSIS AND EFFECT OF TEMPERATURE GRADIENTS
}

\author{
A. Camps \\ Remote Sensing Lab (RSLAB), Dep. of Signal Theory and Communications (TSC), \\ Universitat Politècnica de Catalunya (UPC) Campus Nord, D4-016; 08034 Barcelona, Spain \\ and IEEC-CRAE/UPC. \\ Email: camps@tsc.upc.edu, Phone: +34 934054153, Fax: +34 934017232
}

\begin{abstract}
The performance analysis of microwave radiometers is often simplified assuming that most/all circuits are perfectly matched and that all are at the same physical temperature. However, the increasing performances demanded to future instruments makes necessary to assess their performance including all these effects: actual complex S-parameters of the different subsystems (non-zero insertion losses, finite matching, finite isolation....), physical temperature gradients etc. in view to devise a software correction, when it is not possible to warrant them by design. A full noisewave analysis is presented in this paper.
\end{abstract}

Index Terms - Microwave radiometers, thermal gradient, noise waves, performance

\section{INTRODUCTION}

The accurate performance of any microwave radiometer can only be assessed by using a detailed noise wave analysis, instead of the simplistic approach consisting of using only transmission Sparameters (insertion losses and isolations) to estimate radiometer's output to input noise temperatures. Noise wave analysis accounts for all reflections within the switching network, noise sources, loads, antennas, amplifiers etc., as well as the internally-generated noise due to network losses.

Figure 1 shows a typical microwave radiometer used in radar altimetry. It includes a 6 switches assembly: 4 to commute between the main antenna and the sky horn, and between the nominal and redundant receivers, and 2 more to act as Dicke switches in both the nominal and redundant receiving chains.

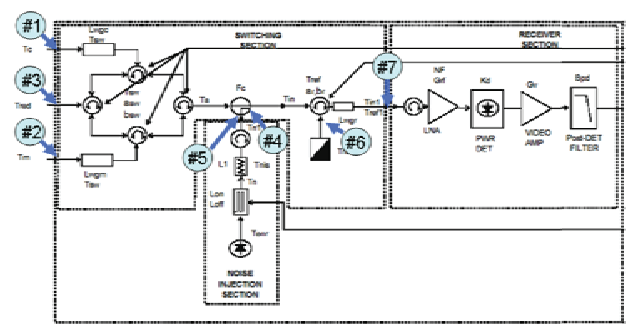

Fig. 1. Microwave radiometer topology.
Due to the symmetry between nominal and redundant receivers, in this analysis only the case in which the nominal receiver is ON, while the redundant one is OFF will be considered. Four different states have to be considered:

- $\quad$ Input $=$ Sky horn $\left(\right.$ antenna temperature $=2.7 \mathrm{~K}^{1}$ ) or Main antenna (antenna temperature between 30 and $315 \mathrm{~K}$ ), and

- Dicke switch (nominal receiver) connecting to antenna or to the reference load.

Figure 1 shows the 7 ports defined at the outputs of the switching section:

- Port 1 corresponds to the sky horn antenna, used for calibration purposes,

- $\quad$ Port 2 corresponds to the main antenna (looking to the Earth),

- $\quad$ Port 3 corresponds to the input of the branch of the switching section + noise injection of the redundant receiver,

- $\quad$ Port 4 corresponds to the internal load within the coupler used to inject noise,

- Port 5 corresponds to the port of the coupler where noise is actually being injected (if noise source is OFF, the radiometer behaves as a Dicke radiometer),

- Port 6 corresponds to the internal load used to match one of the ports of the latching circulator used to implement the Dicke switch, and

- Port 7 corresponds to the input of the nominal receiver.

Note that ports $\# 4$ and $\# 6$ are internally terminated with matched loads that also generate thermal noise. Therefore, they have also been considered in this analysis.

\section{NOISE WAVE ANALYSIS}

A linear $\mathrm{N}$-port can be represented by its $\mathrm{N} \times \mathrm{N}$ scattering matrix (S) and by a $\mathrm{N} \times 1$ noise vector (n) that accounts for the noise waves generated internally [1]:

$$
\mathbf{b}=\mathbf{S} \cdot \mathbf{a}+\mathbf{n},
$$

where $\mathbf{a}$ and $\mathbf{b}$ are the usual incident and outgoing $\mathrm{N} \times 1$ wave vectors defined over a $1 \mathrm{~Hz}$ bandwidth (Fig. 2). In what follows, it will also be assumed that the noise waves are normalized by the square root of the Boltzmann's constant so that the rms value has units of Kelvin.

\footnotetext{
${ }^{1}$ Ohmic losses in the waveguide connecting the sky horn to the radiometer front end increase this value to $\sim 32 \mathrm{~K}$.
} 


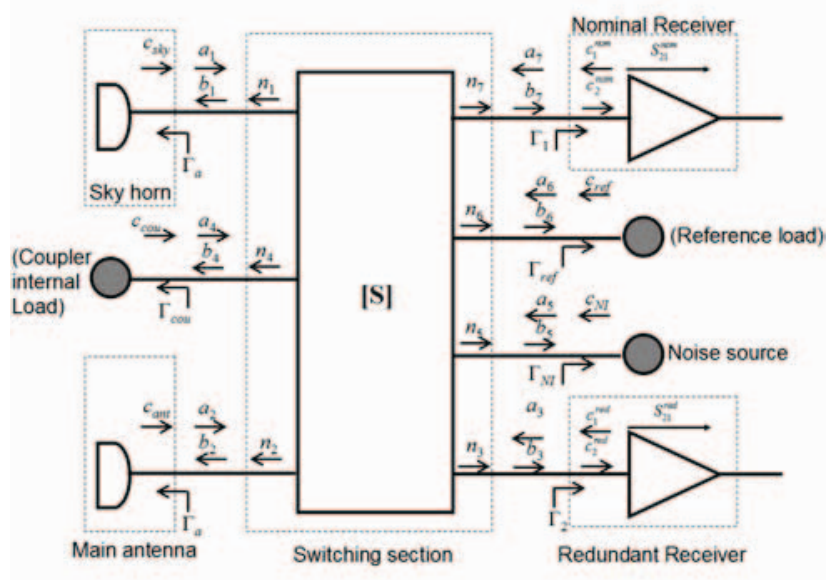

Fig. 2. Noise wave analysis

The incident wave vector can also be written as [2]:

$$
\mathbf{a}=\boldsymbol{\Gamma} \cdot \mathbf{b}+\mathbf{a}_{\mathrm{s}},
$$

where $\mathbf{a}_{\mathbf{s}}$ is the vector of source waves connected to each of the $\mathrm{N}$ ports. In our case (Fig. 2):

$$
\mathbf{a}_{\mathbf{s}}=\left[\begin{array}{lllllll}
c_{s k y} & c_{\text {ant }} & c_{1}^{\text {red }} & c_{\text {cou }} & c_{N I} & c_{r e f} & c_{1}^{\text {nom }}
\end{array}\right]^{T},
$$

whose elements are:

- $\quad c_{s k y}$ is the noise collected by the sky horn antenna from the sky,

- $\quad c_{a n t}$ is the noise collected by the main antenna from the Earth,

- $\quad c_{1}^{\text {red }}$ is the noise generated by the redundant receiving chain towards the input,

- $\quad c_{c o u}$ is the noise generated by the coupler's internal matched load,

- $c_{N I}$ is the noise injected through the coupler,

- $\quad c_{r e f}$ is the noise generated by the reference load, and

- $\quad c_{1}^{\text {nom }}$ is the noise generated by the nominal receiving chain towards the input.

On the other hand $\Gamma$ is a $\mathrm{N} \times \mathrm{N}$ diagonal matrix whose elements are the reflection coefficients of each of the ports as seen from the network (Fig. 2):

$$
\Gamma=\operatorname{diag}\left(\left[\begin{array}{lllllll}
\Gamma_{s k y} & \Gamma_{\text {ant }} & \Gamma_{\text {red }} & \Gamma_{\text {cou }} & \Gamma_{N I} & \Gamma_{\text {ref }} & \Gamma_{R}
\end{array}\right]\right) .
$$

In (4) "diag" means a diagonal matrix with the element vector along its main diagonal. Substituting (2) in (1) and isolating $\mathbf{b}$, the following expression is readily obtained:

$$
\mathbf{b}=(\mathbf{I}-\mathbf{S} \cdot \boldsymbol{\Gamma})^{-1} \cdot \mathbf{S} \cdot \mathbf{a}_{\mathbf{s}}+(\mathbf{I}-\mathbf{S} \cdot \boldsymbol{\Gamma})^{-1} \cdot \mathbf{n},
$$

and defining

$$
\mathbf{\Lambda} \hat{=}(\mathbf{I}-\mathbf{S} \cdot \boldsymbol{\Gamma})^{-1},
$$

as in [3], eqn. (5) can be re-written in a more compact notation as:

$$
\mathbf{b}=\boldsymbol{\Lambda} \cdot \mathbf{S} \cdot \mathbf{a}_{\mathrm{s}}+\boldsymbol{\Lambda} \cdot \mathbf{n} \text {. }
$$

Finally, to take into account the equivalent noise waves $c_{2}^{i}$ (Fig. 2) at the amplifiers' input, the $\mathbf{b}^{\prime}$ vector is defined:

$$
\mathbf{b}^{\prime}=\mathbf{b}+\mathbf{c},
$$

in which $\mathbf{c}$ is defined as:

$$
c=\left[\begin{array}{lllllll}
0 & 0 & 0 & 0 & 0 & 0 & c_{2}^{\text {nom }}
\end{array}\right]^{T} .
$$

In deriving (9) it has been assumed that the redundant channel is off, and no additional noise is generated except for the one generated by the input isolator, whose contribution is given by $c_{1}^{\text {red }}$ in (3).

Radiometer's output is the power of the noise waves at the receivers' output:

$$
P=C_{d} \cdot\left|S_{21}^{\text {nom }}\right|^{2} \cdot\left\langle\left|b_{\text {nom }}^{\prime}\right|^{2}\right\rangle,
$$

where $C_{d}$ is the constant of the detector diode $C_{d}[\mathrm{~V} / \mathrm{W}], S_{21}^{\text {nom }}$ is the $S_{21}$ parameter of the nominal receiving chain $\left(G=\left|S_{21}^{\text {nom }}\right|^{2}\right)$, both subject to thermal drifts. This analysis will be performed in terms of equivalent input noise temperatures and only the $\left\langle\left|b_{\text {nom }}^{\prime}\right|^{2}\right\rangle$ term will be accounted for. In order to compute $\left\langle\left|b_{n o m}^{\prime}\right|^{2}\right\rangle$, the correlation matrix $\mathrm{N}$ of vector $\mathbf{b}^{\prime}$ must be first computed:

$$
\begin{aligned}
\mathbf{N} & =\left\langle\mathbf{b}^{\mathbf{\prime}} \cdot \mathbf{b}^{\mathbf{H}^{\mathbf{H}}}\right\rangle=\left\langle\left(\boldsymbol{\Lambda} \cdot \mathbf{S} \cdot \mathbf{a}_{\mathrm{s}}+\boldsymbol{\Lambda} \cdot \mathbf{n}+\mathbf{c}\right) \cdot\left(\boldsymbol{\Lambda} \cdot \mathbf{S} \cdot \mathbf{a}_{\mathrm{s}}+\boldsymbol{\Lambda} \cdot \mathbf{n}+\mathbf{c}\right)^{\mathbf{H}}\right\rangle= \\
= & \boldsymbol{\Lambda} \cdot \mathbf{S} \cdot\left\langle\mathbf{a}_{\mathbf{s}} \cdot \mathbf{a}_{\mathbf{s}}^{\mathbf{H}}\right\rangle \cdot \mathbf{S}^{\mathbf{H}} \cdot \boldsymbol{\Lambda}^{\mathbf{H}}+\boldsymbol{\Lambda} \cdot \mathbf{S} \cdot\left\langle\mathbf{a}_{\mathrm{s}} \cdot \mathbf{c}^{\mathbf{H}}\right\rangle+\boldsymbol{\Lambda} \cdot\left\langle\mathbf{n} \cdot \mathbf{n}^{\mathbf{H}}\right\rangle \cdot \boldsymbol{\Lambda}^{\mathbf{H}}+ \\
& +\left\langle\mathbf{c} \cdot \mathbf{a}_{\mathrm{s}}^{\mathbf{H}}\right\rangle \cdot \mathbf{S}^{\mathbf{H}} \cdot \boldsymbol{\Lambda}^{\mathbf{H}}+\left\langle\mathbf{c} \cdot \mathbf{c}^{\mathbf{H}}\right\rangle .
\end{aligned}
$$

In evaluating (11), the correlation matrices of the different signals involved must be first computed. The noise matrix of the incoming noise waves is a diagonal one, since the noise coming from the antenna, and the noise waves generated by the receivers towards the input are uncorrelated:

$$
\left\langle\mathbf{a}_{\mathbf{s}} \cdot \mathbf{a}_{\mathbf{s}}^{\mathbf{H}}\right\rangle=\operatorname{diag}\left(\left[\begin{array}{lllllll}
T_{s k y} & T_{A} & T_{r e d} & T_{c o u} & T_{N} & T_{r e f} & T_{n o m}
\end{array}\right]\right),
$$

in which it has been assumed that the physical temperature of the matched load inside the coupler $\left(T_{\text {cou }}\right)$, in the reference load $\left(T_{\text {ref }}\right)$, and of the isolators of the nominal $\left(T_{\text {nom }}\right)$ and redundant $\left(T_{\text {red }}\right)$ receiving chains may be different, and different from the physical temperature of the switching assembly network $\left(T_{p h}\right)$.

In a similar way:

$$
\begin{aligned}
\left\langle\mathbf{a}_{\mathbf{s}} \cdot \mathbf{c}^{\mathbf{H}}\right\rangle= & {\left[\begin{array}{lllllll}
c_{s k y} & c_{\text {ant }} & c_{1}^{\text {red }} & c_{\text {cou }} & c_{N I} & c_{\text {ref }} & c_{1}^{\text {nom }}
\end{array}\right]^{T} . } \\
& {\left[\begin{array}{lllllll}
0 & 0 & 0 & 0 & 0 & 0 & c_{2}^{\text {nom }}
\end{array}\right]=\overline{=} }
\end{aligned},
$$

since there is an isolator before the RF LNA,

$$
\begin{aligned}
& \left\langle\mathbf{c} \cdot \mathbf{a}_{\mathbf{s}}^{\mathbf{H}}\right\rangle=\mathbf{=}, \\
\left\langle\mathbf{c} \cdot \mathbf{c}^{\mathbf{H}}\right\rangle= & {\left[\begin{array}{lllllll}
0 & 0 & 0 & 0 & 0 & 0 & c_{2}^{\text {nom }}
\end{array}\right]^{T} . } \\
& {\left[\begin{array}{llllllll}
0 & 0 & 0 & 0 & 0 & 0 & c_{2}^{\text {nom }}
\end{array}\right]=, } \\
= & \operatorname{diag}\left(\left[\begin{array}{lllllll}
0 & 0 & 0 & 0 & 0 & 0 & T_{R}
\end{array}\right]\right)
\end{aligned}
$$

and [1]:

$$
\left\langle\mathbf{n} \cdot \mathbf{n}^{\mathbf{H}}\right\rangle=T_{p h}\left(\mathbf{I}-\mathbf{S} \cdot \mathbf{S}^{\mathbf{H}}\right) .
$$

In the previous equations: $T_{S k y}$ is the antenna temperature measured by the sky horn $\left(=2.7 \mathrm{~K}^{1}\right), T_{A}$ is the antenna temperature measured by the main antenna $(30-315 \mathrm{~K})$, and $T_{N}$ is the equivalent noise temperature injected through the coupler:

$$
\begin{gathered}
T_{N I^{\prime}}=\left(10^{E N R / 10}-1\right) T_{0}, \\
T_{N}=T_{N I^{\prime}} \cdot \frac{1}{L_{\text {ON } / \text { OFF }} \cdot L_{1}}+T_{p h}\left(1-\frac{1}{L_{\text {ON } / \text { OFF }} \cdot L_{1}}\right),
\end{gathered}
$$

and $T_{R}$ is the receiver equivalent noise temperature [3]. Note that in (18) it has been assumed a perfect matching of the noise injection section. In case of imperfect matching, $T_{N}$ should be replaced by the exact equation providing the amount of noise being actually injected. 


$$
\begin{aligned}
& \text { Substituting eqns. (12)-(16) in (11): } \\
& \mathbf{N}=\mathbf{\Lambda} \cdot \mathbf{S} \cdot \operatorname{diag}\left(\left[\begin{array}{lllllll}
T_{\text {sky }} & T_{A} & T_{\text {red }} & T_{\text {cou }} & T_{N} & T_{\text {ref }} & T_{\text {nom }}
\end{array}\right]\right) \cdot \mathbf{S}^{\mathbf{H}} \cdot \mathbf{\Lambda}^{\mathbf{H}} \\
& +T_{p h} \cdot \mathbf{\Lambda} \cdot\left(\mathbf{I}-\mathbf{S} \cdot \mathbf{S}^{\mathbf{H}}\right) \cdot \mathbf{\Lambda}^{\mathbf{H}}+\operatorname{diag}\left(\left[\begin{array}{lllllll}
0 & 0 & 0 & 0 & 0 & 0 & T_{R}
\end{array}\right]\right),
\end{aligned}
$$

from which the radiometer's output power (before the detector diode) can be computed as:

$$
P=\left|S_{21}^{\text {nom }}\right|^{2} \cdot N_{7,7} \text {. }
$$

Note that the last term in eqn. (19) corresponds to the equivalent noise temperature of the receiver section from port \#7 onwards.

The noise temperature at the output of the switching assembly (port 7) is then equal to:

$$
\begin{aligned}
T_{\text {in }}^{m} & =a_{m} \cdot T_{\text {sky }}+b_{m} \cdot T_{A}+c_{m} \cdot T_{\text {red }}+d_{m} \cdot T_{\text {cou }}+e_{m} \cdot T_{N} \\
& +f_{m} \cdot T_{\text {ref }}+g_{m} \cdot T_{\text {nom }}+1 \cdot T_{R}+h_{m} \cdot T_{p h},
\end{aligned}
$$

where the coefficients $a_{m} \ldots h_{m}$ can be obtained from eqn. (20) as the partial derivative of $N_{7,7}$ with respect to each particular variable. Superscript $m$ is used to indicate the different switch assembly configurations:

- $\quad m=1$ : antenna $=$ sky horn + Dicke switch connected to the antenna,

- $\quad m=2:$ antenna $=$ sky horn + Dicke switch connected to the reference load,

- $\quad m=3$ : antenna $=$ main antenna + Dicke switch connected to the antenna, and

- $\quad m=4$ : antenna $=$ main antenna + Dicke switch connected to the reference load,

These coefficients can be obtained either numerically or analytically. In order to obtain a physical understanding of the influence of the different parameters and assess their impact in the radiometer's performance, in the following sections an analytical study is performed. It has been assumed that all ports are reasonable well matched so that $\Gamma<<1$. In these conditions:

$$
\begin{aligned}
& a_{m} \ldots g_{m} \hat{=} \frac{\partial N_{7,7}^{m}}{\partial T_{p}}=\left|\sum_{n=1}^{7} S_{7, n} \cdot S_{n, p} \cdot \Gamma_{n}+S_{7, p}\right|^{2} \\
& \frac{\partial T_{i n}^{m}}{\partial T_{R}}=\frac{\partial N_{7,7}^{m}}{\partial T_{R}}=1 \\
& h_{m} \quad=\frac{\partial N_{7,7}}{\partial T_{p h}}=1-\sum_{n=1}^{7}\left|S_{n, n}\right|^{2} \quad\left(\text { if all } \Gamma_{i}=0\right)
\end{aligned}
$$

Subscript $p$ refers to the port where the input noise temperature is accounted for: sky $(p=1)$, main antenna $(p=2)$, redundant receiver input isolator $(p=3)$, coupler $(p=4)$, noise temperature injected $(p=5)$, reference temperature $(p=6)$, and nominal receiver input isolator $(p=7)$.

Equation (22c) is only valid for $\Gamma_{i}=0$. If not, it will become very lengthy. Numerical results shown in the following section do not include the above approximation.

\section{NUMERICAL RESULTS}

Table 1 shows typical values for the parameters of a microwave radiometer. Table 2 shows the numerical values of the parameters $a_{m} \ldots h_{m}$ (eqns. (22a) and (22c) without approximations) computed from the S-parameters of the whole switching assembly (Fig. 1), for the subsystem parameters with their nominal values (Table 1), and for the 4 possible configurations $(m)$ of the switching assembly. Columns 3-5 show the nominal values of the $a_{m} \ldots h_{m}$ parameters and their sensitivity to the uncertainties in the characterization of the switching assembly S-parameters (ferrite switch insertion losses IL and isolation I, and return losses RL) for the possible configurations: a) antenna $=$ sky horn + Dicke switch connected to the antenna, b) antenna $=$ sky horn + Dicke switch connected to the reference load, c) antenna $=$ main antenna + Dicke switch connected to the antenna, and d) antenna $=$ main antenna + Dicke switch connected to the reference load.

Table 1. Typical values for microwave radiometer parameters.

\begin{tabular}{|l|l|}
\hline Insertion Loss of WG sky horn & $0 \mathrm{~dB}$ \\
\hline Insertion Loss of WG Main Ant. & $0.05 \mathrm{~dB}$ \\
\hline Insertion Loss of single Ferrite $\mathrm{SW}$ & $0.15 \pm 0.05 \mathrm{~dB}$ \\
\hline Isolation of a single Ferrite $\mathrm{SW}$ & $25 \pm 0.3 \mathrm{~dB}$ \\
\hline Return loss of a single Ferrite $\mathrm{SW}$ & $23 \pm 0.3 \mathrm{~dB}$ \\
\hline Physical temp. (min/nom/max) & $268 \ldots 298 \ldots 333 \mathrm{~K} \mathrm{~dB}$ \\
\hline Coupling factor of noise coupler & $15 \mathrm{~dB}$ \\
\hline Directivity of noise coupler & $20 \mathrm{~dB}$ \\
\hline ENR at Noise diode output & $25 \pm 0.01 \mathrm{~dB} /{ }^{\circ} \mathrm{C} \pm 0.12 \mathrm{~dB} /$ year \\
\hline Insertion Loss of PIN switch $\left(\mathrm{L}_{\mathrm{ON}}\right)$ & $1.5 \mathrm{~dB}$ \\
\hline Isolation of PIN switch $\left(\mathrm{L}_{\mathrm{OFF}}\right)$ & $40 \mathrm{~dB}$ \\
\hline
\end{tabular}

Table 2. Numerical values of the parameters $a_{m} \ldots h_{m}$ for different signal paths (a-d: see text for description) and sensitivity to uncertainties in the characterization of Insertion losses (IL), isolation (I) and return losses (RL)

a)

\begin{tabular}{|c|c|c|c|c|}
\hline & Coeffs. & $\Delta$ param $/ \Delta \mathbf{I L} \cdot 10^{-2}$ & $\Delta$ param $/ \Delta \mathbf{I}$ & $\Delta$ param/ $/ \mathbf{R L}$ \\
\hline $\mathbf{a}_{\mathbf{1}}$ & $\mathbf{0 . 8 7 4 6}$ & $\mathbf{0 . 0 0 0 1}$ & $\mathbf{0 . 2 0 1 0}$ & 0.0020 \\
\hline $\mathrm{b}_{1}$ & 0.0003 & 0.0000 & $\mathbf{0 . 0 0 0 0}$ & 0.0000 \\
\hline $\mathrm{c}_{1}$ & 0.0215 & 0.0021 & $\mathbf{0 . 0 0 3 5}$ & 0.0022 \\
\hline $\mathrm{d}_{1}$ & 0.0067 & 0.0002 & $\mathbf{0 . 0 0 1 3}$ & 0.0002 \\
\hline $\mathrm{e}_{1}$ & 0.0317 & 0.0002 & $\mathbf{0 . 0 0 7 0}$ & 0.0002 \\
\hline $\mathrm{f}_{1}$ & 0.0082 & 0.0018 & $\mathbf{0 . 0 0 0 1}$ & 0.0001 \\
\hline $\mathrm{g}_{1}$ & 0.0058 & 0.0002 & $\mathbf{0 . 0 0 0 0}$ & 0.0012 \\
\hline $\mathrm{h}_{1}$ & 0.0512 & 0.0017 & $\mathbf{0 . 1 9 6 7}$ & 0.0011 \\
\hline
\end{tabular}

b)

\begin{tabular}{|c|c|c|c|c|}
\hline & Coeffs. & $\Delta$ param $/ \Delta \mathbf{I L} \cdot 10^{-4}$ & $\Delta$ param $/ \Delta \mathbf{I}$ & $\Delta$ param $/ \Delta \mathbf{R L}$ \\
\hline $\mathbf{a}_{1}$ & 0.0140 & 0.0005 & $\mathbf{0 . 0 0 2 9}$ & 0.0000 \\
\hline $\mathrm{b}_{1}$ & 0.0000 & 0.0012 & $\mathbf{0 . 0 0 0 0}$ & 0.0000 \\
\hline $\mathrm{c}_{1}$ & 0.0002 & 0.1191 & $\mathbf{0 . 0 0 0 0}$ & 0.0000 \\
\hline $\mathrm{d}_{1}$ & 0.0001 & 0.0301 & $\mathbf{0 . 0 0 0 0}$ & 0.0000 \\
\hline $\mathrm{e}_{1}$ & 0.0008 & 0.0277 & $\mathbf{0 . 0 0 0 1}$ & 0.0000 \\
\hline $\mathrm{f}_{1}$ & $\mathbf{0 . 9 5 4 5}$ & 0.0031 & $\mathbf{0 . 2 1 8 2}$ & 0.0015 \\
\hline $\mathrm{g}_{1}$ & 0.0055 & 0.0082 & $\mathbf{0 . 0 0 0 1}$ & 0.0012 \\
\hline $\mathrm{h}_{1}$ & 0.0249 & 0.0003 & $\mathbf{0 . 2 1 3 8}$ & 0.0011 \\
\hline
\end{tabular}

c)

\begin{tabular}{|c|c|c|c|c|}
\hline & Coeffs. & $\Delta$ param $/ \Delta \mathbf{I L}$ & $\Delta$ param $/ \Delta \mathbf{I}$ & $\Delta$ param $/ \Delta \mathbf{R L}$ \\
\hline $\mathbf{a}_{1}$ & 0.0002 & 0.0000 & $\mathbf{0 . 0 0 0 0}$ & 0.0000 \\
\hline $\mathrm{b}_{1}$ & $\mathbf{0 . 8 6 5 4}$ & 0.0001 & $\mathbf{0 . 1 9 8 7}$ & 0.0014 \\
\hline $\mathrm{c}_{1}$ & 0.0211 & $\mathbf{0 . 0 0 2 1}$ & $\mathbf{0 . 0 0 3 4}$ & 0.0000 \\
\hline $\mathrm{d}_{1}$ & 0.0066 & $\mathbf{0 . 0 0 0 2}$ & $\mathbf{0 . 0 0 1 3}$ & 0.0000 \\
\hline $\mathrm{e}_{1}$ & 0.0316 & 0.0002 & $\mathbf{0 . 0 0 7 1}$ & 0.0001 \\
\hline $\mathrm{f}_{1}$ & 0.0082 & $\mathbf{0 . 0 0 1 8}$ & 0.0001 & 0.0000 \\
\hline $\mathrm{g}_{1}$ & 0.0058 & $\mathbf{0 . 0 0 0 2}$ & 0.0000 & 0.0012 \\
\hline $\mathrm{h}_{1}$ & 0.0611 & $\mathbf{0 . 0 0 1 7}$ & $\mathbf{0 . 1 9 4 6}$ & 0.0011 \\
\hline
\end{tabular}

d)

\begin{tabular}{|c|c|c|c|c|}
\hline & Coeffs. & $\Delta$ param $/ \Delta \mathbf{I L} \cdot 10^{-4}$ & $\Delta$ param $/ \Delta \mathbf{I}$ & $\Delta$ param $/ \Delta \mathbf{R L}$ \\
\hline $\mathbf{a}_{\mathbf{1}}$ & 0.0000 & 0.0008 & $\mathbf{0 . 0 0 0 0}$ & 0.0000 \\
\hline $\mathrm{b}_{1}$ & 0.0140 & 0.0005 & $\mathbf{0 . 0 0 2 9}$ & 0.0000 \\
\hline $\mathrm{c}_{1}$ & 0.0001 & 0.0731 & $\mathbf{0 . 0 0 0 0}$ & 0.0000 \\
\hline $\mathrm{d}_{1}$ & 0.0049 & 0.1108 & $\mathbf{0 . 0 0 1 1}$ & 0.0000 \\
\hline $\mathrm{e}_{1}$ & 0.0007 & 0.2938 & $\mathbf{0 . 0 0 0 1}$ & 0.0000 \\
\hline $\mathrm{f}_{1}$ & $\mathbf{0 . 9 5 4 4}$ & 0.0031 & $\mathbf{0 . 2 2 1 2}$ & 0.0016 \\
\hline $\mathrm{g}_{1}$ & 0.0055 & 0.0082 & $\mathbf{0 . 0 0 0 1}$ & 0.0012 \\
\hline $\mathrm{h}_{1}$ & 0.0204 & 0.0003 & $\mathbf{0 . 2 1 6 8}$ & 0.0011 \\
\hline
\end{tabular}

Radiometer's output in NIR mode $\left(T_{A}<T_{r e f}\right)$ is obtained from the balance equation of the noise temperature at the output of the switching assembly during two halves of the Dicke switching period. During a fraction $(1-\eta)$ of the first half, the radiometer is 
looking at the antenna (either the sky horn or the main antenna). During the remaining fraction $(\eta)$ the radiometer is looking at the antenna and noise is injected. During the second half the radiometer is looking at the reference load. In the following sections the radiometer outputs are derived from the balance equations when looking to the sky horn and the main antenna.

NIR Balance equation when looking to the sky horn: The NIR balance equation when looking to the sky horn is given by substituting the $a_{1} \ldots h_{1}$ parameters when looking to the antenna and the $a_{2} \ldots h_{2}$ parameters when looking to the reference load (eqn. (21)):

$$
\left.(1-\eta) \cdot T_{\text {in }}^{m=1}\right|_{T_{N, \text { OFF }}}+\left.\eta \cdot T_{\text {in }}^{m=1}\right|_{T_{N, \text { ON }}}=\left.T_{\text {in }}^{m=2}\right|_{T_{N, \text { OFF }}}
$$

from which the NIR observable (duty cycle of the injected pulse) can be derived:

$$
\begin{aligned}
\eta & =\frac{1}{e_{1} \cdot\left(T_{N, O N}-T_{N, O F F}\right)}\left\{\left(a_{2}-a_{1}\right) \cdot T_{\text {sky }}+\left(b_{2}-b_{1}\right) \cdot T_{A}+\right. \\
& +\left(c_{2}-c_{1}\right) \cdot T_{\text {red }}+\left(d_{2}-d_{1}\right) \cdot T_{\text {cou }}+\left(e_{2}-e_{1}\right) \cdot T_{N, \text { OFF }}+ \\
& \left.+\left(f_{2}-f_{1}\right) \cdot T_{\text {ref }}+\left(g_{2}-g_{1}\right) \cdot T_{\text {nom }}+\left(h_{2}-h_{1}\right) \cdot T_{p h}\right\},
\end{aligned}
$$

Isolating $T_{s k y}$ in eqn. (24), the sky temperature can be derived:

$$
\begin{aligned}
T_{\text {shy }}= & \frac{b_{2}-b_{1}}{a_{1}-a_{2}} \cdot T_{A}+\frac{c_{2}-c_{1}}{a_{1}-a_{2}} \cdot T_{\text {red }}+\frac{d_{2}-d_{1}}{a_{1}-a_{2}} \cdot T_{\text {cou }}+\frac{e_{2}}{a_{1}-a_{2}} \cdot T_{N, \text { OFF }}+ \\
- & \frac{e_{1}}{a_{1}-a_{2}} \cdot \eta \cdot\left(T_{N, O N}-T_{N, O F F}\right)+\frac{f_{2}-f_{1}}{a_{1}-a_{2}} \cdot T_{\text {ref }}+\frac{g_{2}-g_{1}}{a_{1}-a_{2}} \cdot T_{\text {nom }}+\frac{h_{2}-h_{1}}{a_{1}-a_{2}} \cdot T_{p h}(25) \\
\approx & (0.0371 \pm 0.0036) \cdot \eta \cdot\left(T_{N, O N}-T_{N, O F F}\right)+ \\
& (1.0244 \pm 0.1507) \cdot T_{p h}+1.1044 \cdot \Delta T_{\text {ref }}-0.0003 \cdot T_{A}
\end{aligned}
$$

The numerical values above are derived from Table 2 by performing 10.000 Montecarlo simulations, from which the mean and the standard deviations are computed. Equation (25) shows the impact in the measured antenna temperature (sky horn) due to temperature gradients within the assembly and the leakage of the main antenna. Assuming that the physical temperature of the matched load inside the coupler $\left(T_{\text {cou }}\right)$, in the reference load $\left(T_{\text {ref }}\right)$, and of the isolators of the nominal $\left(T_{\text {nom }}\right)$ and redundant $\left(T_{\text {red }}\right)$ receiving chains are equal to the physical temperature of the assembly switching network $\left(T_{p h}\right)$, the sky temperature is given by: $T_{\text {sky }} \approx(0.0371 \pm 0.0036) \cdot \eta \cdot\left(T_{N, \text { ON }}-T_{N, \text { OFF }}\right)+(1.0244 \pm 0.1507) \cdot T_{p h}$, (26) that, as expected, presents a sensitivity to the physical temperature equal to $\partial T_{A}^{N I R} / \partial T_{p h}=1.02 \mathrm{~K} /{ }^{\circ} \mathrm{C}$. Obviously, this value is driven by the coefficient of the temperature of the reference load.

NIR Balance equation when looking to the main antenna: The NIR balance equation when looking to the main antenna is obtained in a similar way by substituting the $a_{3} \ldots h_{3}$ parameters when looking to the antenna and the $a_{4} \ldots h_{4}$ parameters when looking to the reference load, from which $T_{A}$ can be derived as:

$$
\begin{aligned}
T_{A} \quad= & \frac{a_{4}-a_{3}}{b_{3}-b_{4}} \cdot T_{s k y}+\frac{c_{4}-c_{3}}{b_{3}-b_{4}} \cdot T_{\text {red }}+\frac{d_{4}-d_{3}}{b_{3}-b_{4}} \cdot T_{\text {cou }}+\frac{e_{4}}{b_{3}-b_{4}} \cdot T_{N, O F F}+ \\
& -\frac{e_{3}}{b_{3}-b_{4}} \cdot \eta \cdot\left(T_{N, O N}-T_{N, O F F}\right)+\frac{f_{4}-f_{3}}{b_{3}-b_{4}} \cdot T_{r e f}+\frac{g_{4}-g_{3}}{b_{3}-b_{4}} \cdot T_{\text {nom }}+\frac{h_{4}-h_{3}}{b_{3}-b_{4}} \cdot T_{p h} \\
\approx & -(0.0373 \pm 0.0036) \cdot \eta \cdot\left(T_{N, O N}-T_{N, O F F}\right)+ \\
& +(1.0417 \pm 0.1505) \cdot T_{p h}+1.1159 \cdot \Delta T_{\text {ref }}
\end{aligned}
$$

The numerical values above are also derived from Table 2 and performing 10.000 Montecarlo simulations. If all temperatures are the same, the antenna temperature simplifies to:

$$
T_{A} \approx-(0.0373 \pm 0.0036) \cdot \eta \cdot\left(T_{N, O N}-T_{N, O F F}\right)+(1.0417 \pm 0.1505) \cdot T_{p h}, \text { (30) }
$$

that, as expected, presents a sensitivity to the physical temperature close to the theoretical value of $1 \mathrm{~K} /{ }^{\circ} \mathrm{C}: \partial T_{A}^{N I R} / \partial T_{p h}=1.04 \mathrm{~K} /{ }^{\circ} \mathrm{C}$. These coefficients, including that of the injected noise term are the same as in eqn. (26), within the numerical round-off error (2-4\%).

In eqns. (25) and (29) $\Delta T_{p}$ are the differences between the temperatures of port $p\left(T_{p}\right)$ and the physical temperature of the switching assembly $\boldsymbol{T}_{p h}$, that must be precisely monitored. As intuitively expected, these results show that the most important temperature gradient is that of the reference load, which drives the coefficient of the drift in the measured antenna temperature. Gradients in the switching assembly induce an error $\sim 1.1 \cdot \Delta T_{\text {ref }}$.

\section{CONCLUSIONS}

Theoretical analyses and simulation results have been presented for a Noise Injection Radiometer with typical subsystem values and their measured uncertainties, such as insertion losses (IL), matching (RL), and isolation (I). To simplify the mathematical formulation and obtain closed-form expressions, it has been assumed that IL $\sim 1$, and $\mathrm{RL}, \mathrm{I}<<0$, which is very often the case. The analytical expressions for the case of having a temperature gradient approaching to zero tend to the well-known expressions found in most text books when everything is assumed to be at the same physical temperature $T_{\text {ph }}$. In case the thermal gradient is not zero, terms $\sim 1.1 \cdot \Delta T_{r e f}$. appear in the general equations.

\section{ACKNOWLEDGEMENTS}

This work has been partially supported by the Spanish Ministry of Education and Culture CICYT AYA2008-05906-C02-01/ESP

\section{REFERENCES}

[1] S.W. Wedge and D.B. Rutledge, Wave techniques for noise modeling and measurement, IEEE Transactions On Microwave Theory and Techniques, Vol. 40(11), pp. 2004-2012, November 1992.

[2] J. Randa, Noise characterization of multiport amplifiers, IEEE Transactions on Microwave Theory and Techniques, Vol. 49, pp. 1757-1763, 2001.

[3] I. Corbella, F. Torres, A. Camps, N. Duffo, M. Vall-llossera, K. Rautiainen, M. Martín-Neira, and A. Colliander, Analysis of Correlation and Total Power Radiometer Front-Ends Using Noise Waves, IEEE Transactions on Geoscience and Remote Sensing, Vol. 43(11), 2459-2452, November 2005. 